\title{
Técnicas em cirurgia bariátrica: uma revisão da literatura
}

\author{
Bariatric surgery techniques: a literature review
}

Jorge Luiz de Mattos Zeve ${ }^{1}$, Poliana Oliveira Novais ${ }^{2}$, Nilvan de Oliveira Júnior ${ }^{2}$

\section{RESUMO}

Introdução: A obesidade é uma condição clínica crônica de etiologia multifatorial cujo fracasso no tratamento clínico e terapêutico levam a necessidade de uma intervenção mais eficaz, a cirúrgica. As cirurgias bariátricas podem modificar apenas o estômago, a fim de reduzir o espaço para o alimento e promover a sensação de saciedade, ou em complemento o intestino delgado, visando uma diminuição do local para absorção de nutrientes.

Objetivo: Revisar as técnicas de intervenção em cirurgia bariátrica utilizadas no mundo atual, no intuito de compreender as decisões do cirurgião no que diz respeito à escolha das mesmas, tendo por base comparações dessas técnicas.

Materiais e Métodos: Realizou-se estudo exploratório tipo revisão bibliográfica nas bases de dados SciELO, LILACS, PubMed, Bireme, sob os seguintes indexadores: técnicas em cirurgia bariátrica, derivação biliopancreática, gastroplastia vertical com bandagem, banda gástrica ajustável, gastrectomia vertical, cirurgia de derivação gástrica, derivações gástricas em Y de Roux, derivação biliopancreática com gastrectomia vertical e preservação pilórica.

Resultados: De 66 referências relativas às técnicas em cirurgia bariátrica analisadas, 40 foram selecionadas entre capítulos de obras bibliográficas, consensos, revisões sistemáticas, meta-análises, estudos clínicos randomizados e controlados. Foram revisadas técnicas cirúrgicas para tratamento da obesidade classificadas como: restritivas, predominantemente restritivas e mal absortivas.

Conclusão: Atualmente, diferentes técnicas cirúrgicas no tratamento da obesidade são reconhecidas e recomendadas no mundo inteiro. A escolha da técnica pelo cirurgião irá se basear em informações sobre seu mecanismo de funcionamento, resultados e riscos, que podem ser obtidas através de relevantes publicações científicas.

Palavras-chave: cirurgia bariátrica; obesidade; perda de peso.

\begin{abstract}
Introduction: Obesity is a chronic medical condition of multifactorial etiology whose failure in clinical and therapeutic treatment leads to the need of more effective intervention, the surgical procedure. The bariatric surgeries may only change the stomach in order to reduce the space for the food and promote the feeling of fullness, or in addition may change the small intestine, in order to place a decrease in absorption of nutrients.

Objective: To review the intervention techniques in bariatric surgery, in order to understand the reasons why surgeons choose a specific technique.

Materials and Methods: We conducted an exploratory study trough a bibliographic review in SciELO, LILACS, PubMed and BIREME databases, under the following indexes: bariatric surgery techniques, biliopancreatic diversion, vertical banded gastroplasty, adjustable gastric band, vertical gastrectomy, gastric bypass surgery, Roux-en-Y gastric bypass, sleeve gastrectomy with duodenal switch.

Results: From 66 references related to techniques in bariatric surgery analyzed, 40 were selected among bibliographic work chapters, consensus, systematic reviews, meta-analysis, randomized and controlled clinical trials. We reviewed the surgical techniques for the treatment of obesity classified as restrictive, predominantly restrictive and poorly absorptive.

Conclusions: Currently, different surgical techniques in the treatment of obesity are recognized and recommended worldwide. The surgeon's choice for a technique will be based on information about its mechanism, performance and risks that can be found on relevant scientific publications.
\end{abstract}

Keywords: bariatric surgery; obesity; weight loss.

\footnotetext{
${ }^{1}$ Médico. Doutor em Ciências da Saúde pela Universidade de Brasília (UnB). Professor Assistente de Cirurgia do Curso de Medicina da Universidade Federal do Tocantins (UFT).

${ }^{2}$ Acadêmicos. Curso de Medicina da UFT.
} 


\section{INTRODUÇÃo}

A obesidade é uma condição clínica crônica, de etiologia multifatorial, cujo tratamento envolve várias abordagens, tais como nutricional, medicamentosa e prática de exercícios físicos ${ }^{1,2}$. No entanto, vários pacientes não respondem às manobras clínicas e terapêuticas, necessitando de uma intervenção mais eficaz, a cirúrgica.

A intervenção cirúrgica no tratamento da obesidade teve seus primeiros estudos na década de 50. As primeiras técnicas consistiam em criar um grande efeito disabsortivo, efetuando a exclusão de grande parte do intestino delgado ${ }^{2,3}$. Em 1986, Fobi propôs um bypass gástrico utilizando anel de silicone sem incisões no estômago, sendo o anel utilizado como elemento de limitação de distensão da bolsa ${ }^{3}$. Na década de 1990 , Capella propôs uma operação similar à de Fobi a qual realizava uma gastroplastia associada a um bypass gastrointestinal. Tais procedimentos vêm trazendo dimensões menores ao reservatório. E são estas modificações, no bypass gástrico, realizadas por Fobi e Capella, consideradas hoje o "padrão ouro" da cirurgia bariátrica $^{2}$. A partir do exposto, pode-se entender a cirurgia bariátrica como o nome científico para o tratamento cirúrgico da obesidade, sendo, portanto, um termo que engloba todos os tipos de procedimentos cirúrgicos que visam à indução de redução de massa corpórea em pacientes obesos ${ }^{4}$.

A cirurgia enquanto tratamento da obesidade deve ser cogitada em pacientes que já tiveram diversas tentativas de tratamento convencional (lê-se: clínico), porém não atingiram redução satisfatória e sustentada de peso ${ }^{5}$ (mais de $5 \%$ do peso corporal mantido por mais de cinco anos). Se tratando de um procedimento cirúrgico de grande porte em pacientes que apresentam riscos aumentados de complicações, é imprescindível que a seleção seja cuidadosa e embasada em critérios que avaliem os riscos e benefícios da intervenção cirúrgica ${ }^{5}$.

Segundo o Consenso Bariátrico definido pela Sociedade Brasileira de Cirurgia Bariátrica e Metabólica $^{6}$, as cirurgias bariátricas, independentemente da técnica a ser utilizada, estão indicadas, em relação à massa corpórea, para as pessoas com índice de massa corporal (IMC) $>40 \mathrm{~kg} / \mathrm{m}^{2}$, independentemente da presença de comorbidades (doenças agravadas pela obesidade e que melhoram quando a mesma é tratada de forma eficaz) e IMC entre 35 e $40 \mathrm{~kg} / \mathrm{m}^{2}$ na presença de comorbidade.

As cirurgias bariátricas podem ser divididas em cirurgias restritivas e mistas ${ }^{2,4}$, conforme a tabela 1. As cirurgias restritivas são aquelas onde o único órgão modificado é o estômago e que visam provocar a redução do espaço para o alimento dentro da cavidade gástrica, assim, com uma pequena quantidade de comida o paciente terá a sensação de saciedade ${ }^{4}$. As mais comuns são: gastroplastia vertical com bandagem, balão intragástrico e bandagem gástrica ajustável por vídeo $^{3,4}$. A colocação do balão intragástrico é um procedimento endoscópico ${ }^{7}$, e tem utilização reconhecida como método terapêutico restritivo, utilizado como auxiliar no preparo pré-operatório para outros procedimentos bariátricos. Já nas cirurgias mistas, além do estômago, o intestino do paciente também é alterado ${ }^{2,8}$. Neste grupo, além do fator

TABELA 1 - Técnicas cirúrgicas e suas classificações*

\begin{tabular}{ll}
\hline Classificação & \multicolumn{1}{c}{ Técnica } \\
\hline Restritiva & $\begin{array}{l}\text { Bandagem gástrica } \\
\text { Gastrectomia vertical } \\
\text { Gastrectomia vertical com bandagem } \\
\text { Balão intragástrico }\end{array}$ \\
Predominantemente restritiva & Derivações gástricas em Y de Roux com ou sem anel de contenção \\
Predominantemente mal absortiva & $\begin{array}{l}\text { Derivação biliopancreática com gastrectomia horizontal com ou sem } \\
\text { preservação gástrica distal } \\
\text { Derivação biliopancreática com gastrectomia vertical e preservação } \\
\text { pilórica }\end{array}$ \\
\hline${ }^{*}$ De acordo com a Sociedade Brasileira de Cirurgia Bariátrica e Metabólica. Consenso Bariátrico, 2008
\end{tabular}


restritivo que provoca a sensação de saciedade com uma pequena quantidade de alimento, também existe um fator disabsortivo, o qual é conseguido pela diminuição do local de absorção de nutrientes no intestino delgado. As técnicas mistas mais conhecidas são: derivação biliopancreática com gastrectomia distal $^{9,10}$ (Cirurgia de Scopinaro) e derivação gastrojejunal em Y-de-Roux ${ }^{4,10}$ (Cirurgia de FobiCapella).

Diante da magnitude do tema, o presente artigo teve por objetivo revisar as técnicas de intervenção em cirurgia bariátrica utilizadas no mundo atual, no intuito de compreender as decisões do cirurgião no que diz respeito à escolha das mesmas, tendo por base comparações dessas técnicas.

\section{MATERIAIS E MÉTODOS}

O presente estudo é uma revisão da literatura, realizada no período de 2010 a 2011, englobando estudos que abordam as técnicas em cirurgia bariátrica reconhecidas pelo Conselho Federal de Medicina ${ }^{11}$, compreendidos entre os anos de 1990 e 2011. Para tanto, utilizou-se legislação pertinente, livros, bem como trabalhos científicos divulgados nas bases de dados SciELO, LILACS, PubMed, Bireme.

Foram aplicados indexadores em português (técnicas em cirurgia bariátrica, derivação biliopancreática, gastroplastia vertical com bandagem, banda gástrica ajustável, gastrectomia vertical, cirurgia de derivação gástrica, derivações gástricas em Y-de-Roux, derivação biliopancreática com gastrectomia vertical e preservação pilórica), em inglês (bariatric surgery procedures, biliopancreatic diversion, vertical banded gastroplasty, adjustable gastric band, gastric plication, vertical gastrectomy, vertical sleeve, gastric bypass surgery, roux-en-y gastric by-pass, sleeve gastrectomy with duodenal switch) e em espanhol (procedimientos en cirugía bariátrica, derivación biliopancreática, gastroplastia vertical con banda, banda gástrica ajustable, plicatura gástrica, gastrectomía vertical, vertical en manga, cirugía de bypass gástrico, bypass gástrico en $Y$-de-Roux, gastrectomía en manga con cruce duodenal).

Após a identificação do material que continha algum dos descritores acima relacionados, seguindo um método rigoroso de busca e seleção de pesquisas, prosseguiu-se para avaliação da relevância e validade das pesquisas encontradas, coleta, síntese e interpretação dos dados oriundos dos trabalhos, a fim de se tecer algumas considerações acerca do objeto de estudo desta pesquisa. Foram mantidos estudos que atenderam aos critérios de inclusão: trabalho original, relato de caso, artigo de revisão e meta-análise, publicado no período de 1990 a 2011, contemplando estudos experimentais com humanos, incluindo ensaios clínicos randomizados e controlados.

\section{RESULTADOS E DISCUSSÃO}

Das referências que abordavam as técnicas para a execução da cirurgia bariátrica, 66 foram analisadas, das quais, 26 foram excluídas através das leituras seletiva e analítica por não se adequarem ao objetivo do presente estudo.

\section{Cirurgias restritivas}

\section{Bandagem gástrica ajustável}

A utilização da bandagem gástrica ajustável (figura 1) por via laparoscópica para o tratamento da obesidade mórbida, tal como é descrita atualmente, tem Kuzmak $^{12,13}$ como principal responsável. Foi ele quem idealizou fazê-la ajustável mediante a utilização de um

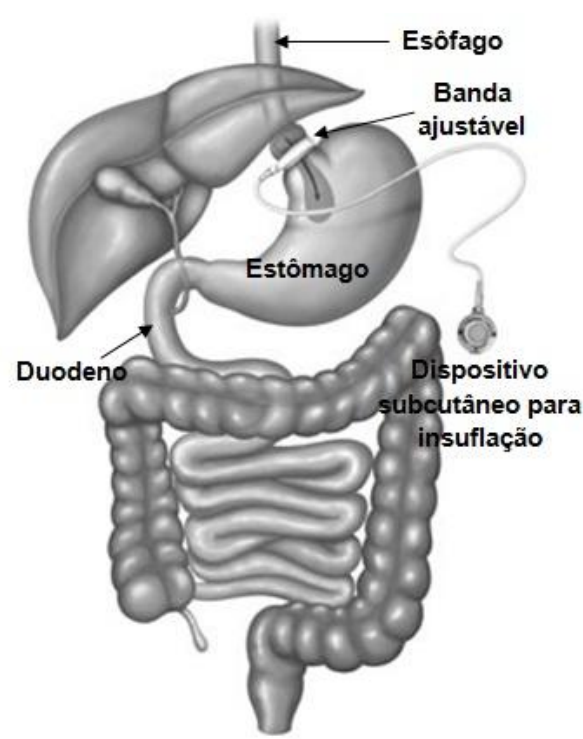

FIGURA 1 - Banda gástrica ajustável.

Fonte: (http://www.sbcb.org.br/cbariatrica.asp?menu=1) 
reservatório que permite sua calibração no pósoperatório, conforme a necessidade do paciente, e também foi quem permitiu a possibilidade de colocá-la por via laparoscópica.

Entretanto, foram Broadbent et al. ${ }^{14}$ no início dos anos 90, os primeiros a realizar uma simples bandagem gástrica por via laparoscópica, e Belachew et al. ${ }^{15}$ os primeiros a utilizar uma banda gástrica ajustável pela mesma via.

A técnica se traduz na introdução de uma prótese de silicone por videolaparoscopia na porção alta do estômago, sendo o diâmetro interno da banda regulado por injeção de líquido no reservatório através de um mecanismo percutâneo de insuflação. O órgão ganha a configuração de uma ampulheta ${ }^{16}$. Diante disso, a distensão gástrica (bolsa gástrica) e esofágica, na presença de alimentos sólidos, emitem para o cérebro, por meio de mecanismos humorais e neurais, sinais que levariam à saciedade.

Essa técnica agrega vários aspectos que podem trazer benefícios em longo prazo para um percentual da população obesa ${ }^{17}$. Diferente das outras técnicas, não envolve a utilização de grampeadores, não há incisão ou abertura do estômago, bem como não há alteração permanente do trato gastrointestinal. Diante disso, caso sua retirada seja necessária, a anatomia gástrica poderá ser restituída. Há ainda a possibilidade do ajuste da banda gástrica, causando maior ou menor dificuldade na ingestão de alimentos pelo paciente, reconhecidamente uma das grandes vantagens desse método ${ }^{18}$.

A média de perda de peso é de, aproximadamente, $35,8 \%$ do peso inicial, em dois anos, sendo que a perda é maior no início e diminui gradualmente ${ }^{17-19}$. No entanto, pode ocorrer o acomodamento dos pacientes para com a restrição gástrica. Estes passam a se alimentar com maior frequência, bem como, com alimentos líquidos e calóricos, o que gera altos índices de falha na perda de peso após dez anos, como apontam alguns estudos ${ }^{18}$. Esta técnica de emagrecimento, segundo Zilberstein et al. ${ }^{19}$, tem sido, inclusive, questionada devido à frequência de reganho de peso.

\section{Gastroplastia vertical com bandagem}

A gastroplastia vertical com bandagem é uma técnica de operação restritiva, simples, rápida, com baixos índices de complicações e mortalidade ${ }^{20}$. Em 1982, esse método foi introduzido e, inicialmente, se baseou no fato de a musculatura da pequena curvatura do estômago ser mais resistente à dilatação. Isso tornou possível a redução gástrica pura e, posteriormente, passou a usar um anel de silicone, prevenindo alargamentos $^{21}$.

O procedimento consiste na realização de uma sutura na região da cárdia, via laparoscopia, originando um pequeno reservatório gástrico, com capacidade de, aproximadamente, $20 \mathrm{ml}$, cujo fluxo é regulado por um anel de polipropileno ${ }^{16,20,21}$. O anel é colocado no orifício de saída, tornando o esvaziamento desta pequena câmara mais lento. Ocorre, portanto, restrição mecânica à ingestão de alimentos e retardo do esvaziamento gástrico, provocando a sensação de saciedade com pequena ingestão alimentar ${ }^{22}$.

Contudo, este procedimento apresenta alta incidência de recidiva da obesidade após 10 anos de seguimento, motivo pelo qual vem sendo abandonado mundialmente ${ }^{24}$. Uma das razões propostas para este fato é o aprendizado realizado pelos pacientes. Eles passam a selecionar e ingerir alimentos líquidos hipercalóricos com uma passagem rápida pelo "estômago estreitado".

Através desse procedimento, os pacientes reduzem, em média, $30 \%$ de seu peso inicial nos primeiros $\operatorname{anos}^{16,24}$. Garrido et al. ${ }^{23}$ observaram que a perda média foi de $19,7 \%$ do peso inicial, após acompanhamento por cinco anos dos pacientes não reoperados por recidiva ou falha da técnica.

\section{Gastrectomia Vertical}

A Gastrectomia vertical, também chamada gastrectomia em manga, gastrectomia longitudinal, ou ainda gastrectomia sleeve (figura 2) foi difundida em 1990 por Marceau et al. ${ }^{25}$, proposta como parte de uma derivação biliopancreática sem gastrectomia distal, preservando o piloro e diminuindo o potencial ulcerogênico. Trata-se de um procedimento cirúrgico 


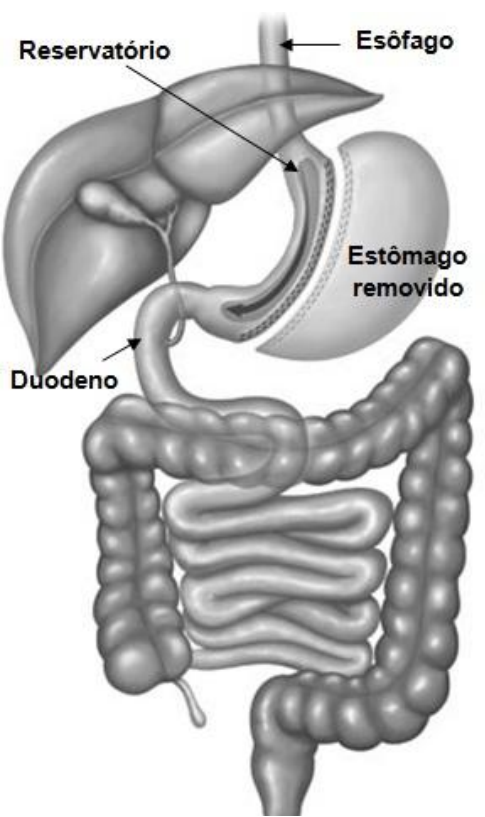

FIGURA 2 - Gastrectomia vertical.

Fonte: (http://www.sbcb.org.br/cbariatrica.asp?menu=1y1)

restritivo aprovado pelo Conselho Federal de Medicina no mês de fevereiro do ano de dois mil e dez ${ }^{11}$.

A literatura recente tem mostrado sua utilização como primeiro tempo cirúrgico em superobesos (IMC > 50 $\mathrm{kg} / \mathrm{m}^{2}$ ) e super superobesos (IMC > $60 \mathrm{~kg} / \mathrm{m}^{2}$ ), objetivando significativa perda de peso com um procedimento de menor porte ${ }^{26,30}$. Assim, o risco cirúrgico torna-se bastante diminuído, e o segundo tempo do tratamento pode ser realizado com melhores condições técnicas e melhor estado clínico do paciente ${ }^{27,28}$.

A gastrectomia vertical funciona como uma restrição gástrica (com remoção de 70 a $80 \%$ do estômago proximal ao antro), com um componente hormonal associado (redução da grelina) ${ }^{29}$.

Dentre as vantagens deste procedimento tem-se a não exclusão do duodeno do trânsito alimentar, portanto, não há interferência com o sítio de absorção de ferro, cálcio, zinco e vitaminas do complexo B. Pode ser transformada, no caso de insucesso, num procedimento com algum componente disabsortivo, como o bypass gástrico em $\mathrm{Y}$ de Roux e a derivação biliopancreática com duodenal switch, e permite acesso às vias biliar e pancreática por métodos endoscópicos habituais $^{31,32}$.

No entanto, trata-se de um método irreversível e

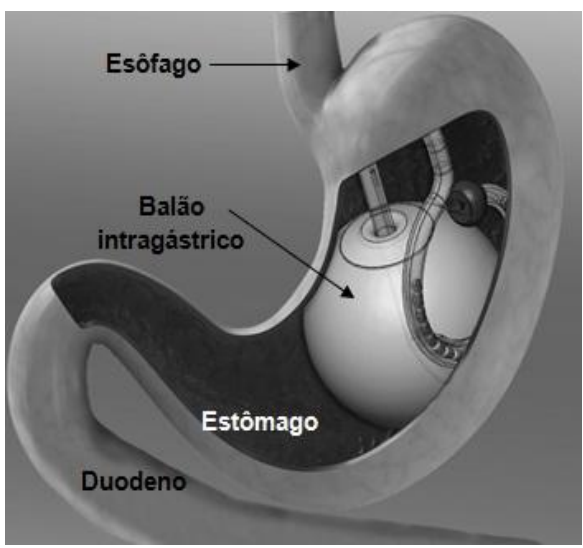

FIGURA 3 - Balão Intragástrico

Fonte:(http://ww1.prweb.com/prfiles/2009/10/17/2278494/Stomachs patz.jpg)

apesar de menor complexidade técnica, pode produzir complicações de alta gravidade e difícil tratamento, como a fístula junto a ângulo de Hiss (esôfago gástrico) e deiscência de sutura gástrica ${ }^{33}$.

Lee et al. ${ }^{33}$ utilizaram a gastrectomia vertical em 216 pacientes e, comparando resultados, concluíram que a técnica é capaz de proporcionar significativa perda de peso quando comparada ao bypass gástrico em $\mathrm{Y}$ de Roux e ao duodenal switch, com morbidade semelhante a lap band, uma das banda gástricas ajustáveis disponíveis. Ainda não existem dados consistentes quanto à sua eficácia em longo prazo na perda e manutenção do peso ${ }^{34}$.

\section{Balões Intragástricos}

Com utilização reconhecida como método terapêutico restritivo, o balão intragástrico (figura 3) é utilizado como auxiliar no preparo pré-operatório para outros procedimentos bariátricos. Tem um papel importante como terapêutica transitória nos doentes com obesidade mórbida e particularmente no subgrupo dos superobesos $^{35}$. Utilizado pela primeira vez em 1985, o balão intragástrico apresentou, inicialmente, inúmeras complicações, tais como obstrução intestinal e hemorragias graves, sendo, inclusive, desconsiderado. Já no fim dos anos oitenta, o aperfeiçoamento de seu material constituinte, levou à criação do modelo de balão utilizado na atualidade ${ }^{36}$.

Trata-se de uma prótese fechada de silicone que é colocada no estômago por via endoscópica, com o 
paciente sob sedação profunda ${ }^{36}$. A prótese se torna esférica após preenchimento com soro fisiológico e azul de metileno. É preenchida até uma capacidade determinada pelo médico gastrenterologista (entre 450 a $700 \mathrm{~mL}$ ) de acordo com a anatomia do doente e mediante a avaliação endoscópica das dimensões e capacidade do lúmen gástrico. O tempo de permanência máximo do balão no estômago é de seis meses e a perda ponderal estimada é de 13 a $20 \mathrm{Kg}$, com redução de 5 a $9 \mathrm{Kg} / \mathrm{m}^{2}$ de $\mathrm{IMC}^{37}$.

Neste contexto, tal terapêutica induz a sensação de plenitude gástrica e de saciedade, visando, sobretudo, perda ponderal pré-cirúrgica para minimizar o risco anestésico-cirúrgico ${ }^{38}$.

\section{Cirurgias mistas}

\section{Predominantemente mal absortivas}

Derivação biliopancreática com gastrectomia horizontal com ou sem preservação gástrica distal

A derivação biliopancreática com gastrectomia horizontal foi descrita em 1976 por Nicola Scopinaro e por isso é denominada Técnica Scopinaro ${ }^{39}$. Ela consiste na redução da capacidade gástrica, eliminação do controle pilórico do esvaziamento gástrico e em má absorção proporcionada pelo bypass da maior parte do intestino delgado, onde são retirados cerca de dois metros e meio de intestino (que, em média, apresenta sete metros).

Com isso, as enzimas que permitem a absorção dos nutrientes passam a ter ação restrita. A remoção do estômago distal reduz o risco de úlcera gástrica. $\mathrm{O}$ paciente passa a necessitar de controle na ingesta de proteínas e cálcio, no intuito de se prevenir doenças ósseas, assim como na ingesta de gorduras, que pioram o odor de seus gases e fezes ${ }^{40}$.

Apesar dos ótimos resultados, o procedimento é complexo. Nicola Scopinaro, em entrevista ao jornal Estadão de São Paulo no ano de 2006, afirma que a técnica exige muito estudo por parte dos cirurgiões e não é todo médico que está habilitado a realizá-la ${ }^{41}$. Ele ressalta: "Ainda assim, hoje, cerca de $20 \%$ das cirurgias bariátricas feitas no mundo são mal absortivas (no Brasil, estima-se que sejam $5 \%$ ). Mas se todos os médicos resolvessem aplicar esta técnica hoje, haveria um massacre de obesos" ${ }^{41}$.

Derivação biliopancreática com gastrectomia vertical e preservação pilórica

Marceau et al. ${ }^{25}$, Baltasar et al. ${ }^{8}$ e Hess et al. ${ }^{42}$, na década de 1990, modificaram a técnica de Scopinaro e desenvolveram a Derivação Biliopancreática com Gastrectomia Vertical e Preservação Pilórica, também conhecida como Técnica Duodenal Switch (figura 4). Ambas as técnicas se diferenciam, uma vez que a Duodenal Switch se traduz em uma gastrectomia realizada no sentido vertical, retirando o fundo, permanecendo pequena parte do antro, e formando um remanescente gástrico em forma de meia lua ${ }^{40}$.

Essa técnica se configura, portanto, em um procedimento de bypass da secreção biliopancreática, que passa a ter contato com os alimentos somente no intestino delgado distal (50 a $70 \mathrm{~cm}$ distante da válvula ileocecal), bem próximo ao intestino grosso. A alça alimentar tem seu tamanho reduzido, passando a medir somente 2,0 a 2,5 metros entre o estômago e o intestino grosso $^{8,25,43}$.

Trata-se de uma técnica pouco restritiva, pois mantêm metade do estômago verticalizado além do piloro, porém é extremamente disabsortiva, o que pode promover casos de diarreia crônica, flatulências, desnutrição e outros problemas.

A técnica Duodenal Switch atualmente vem sendo realizada por vídeocirurgia em alguns centros médicos e seus resultados se assemelham aos da técnica aberta ${ }^{45}$. Faz-se necessário a retirada da vesícula biliar quando se executa esta técnica, pois a maioria dos pacientes apresentará cálculos vesicais futuramente ${ }^{44}$.

\section{Predominantemente restritivas}

Derivações gástricas em Y de Roux, com ou sem anel de contenção

Dentre as técnicas mistas, a derivação gástrica em $Y$ de Roux ou gastroplastia com derivação gastrojejunal em $Y$ de Roux (figura 5), com ou sem anel de contenção, tem destaque devido a sua elevada eficiência e baixa morbimortalidade. Já é considerada a 


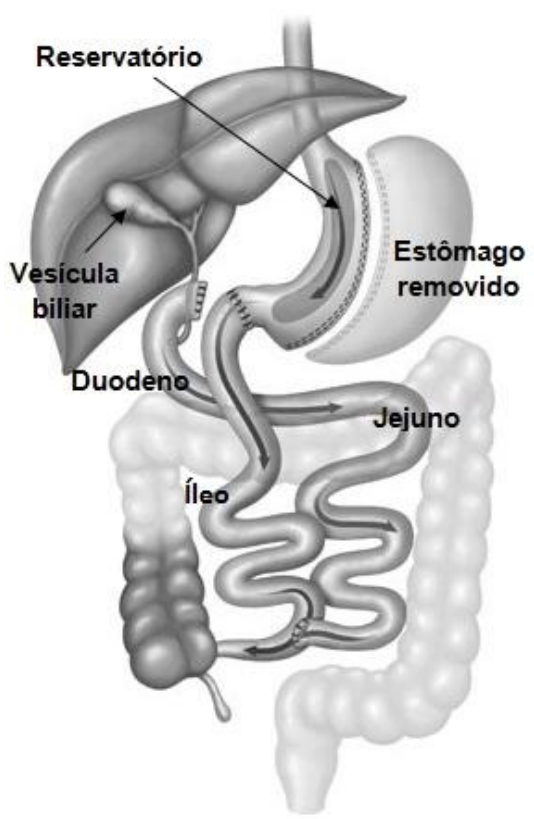

FIGURA 4 - Duodenal switch.

Fonte: (http://www.sbcb.org.br/cbariatrica.asp?menu=1)

técnica mais realizada atualmente no mundo ${ }^{46}$.

Fobi $^{2-3}$, em 1989, e Capella ${ }^{3}$, em 1991, foram os primeiros cirurgiões a descrever essa técnica que, consequentemente, ficou conhecida como Técnica Fobi - Capella. Tal nominação decorre da ampla divulgação deste procedimento, a partir de sua utilização pioneira na disciplina de Cirurgia do Aparelho Digestivo do Hospital das Clínicas da Faculdade de Medicina da Universidade de São Paulo, no início dos anos 90, com os trabalhos de Garrido Júnior ${ }^{47}$.

O método consiste na redução da capacidade gástrica para um volume de aproximadamente $20 \mathrm{ml}$. O estômago remanescente, assim como o duodeno e os primeiros $50 \mathrm{~cm}$ de jejuno, ficam permanentemente excluídos do trânsito alimentar. O pequeno reservatório gástrico é então anastomosado a uma alça jejunal isolada em $\mathrm{Y}$ (daí a origem do nome, sendo Roux o cirurgião criador da técnica) e seu esvaziamento limitado por um anel de silicone, colocado $5,5 \mathrm{~cm}$ distal à transição esofagogástrica e $1,5 \mathrm{~cm}$ proximal à anastomose gastrojejunal, reduzindo o diâmetro da luz gástrica para $12 \mathrm{~mm}$. As secreções provenientes do estômago e do duodeno exclusos desembocam no jejuno por uma anastomose de $100 \mathrm{~cm}$ a $159 \mathrm{~cm}$ abaixo do reservatório, distância que irá depender do IMC do paciente $^{48}$.
Com este procedimento a ingestão de carboidratos simples pode ocasionar a chamada síndrome de dumping ${ }^{49}$, caracterizada por náuseas, vômitos, rubor, dor epigástrica e sintomas de hipoglicemia. Esta síndrome desempenha importante papel na manutenção da perda de peso, porém tende a ser tempo-limitada.

Nota-se a diminuição, no pós-operatório, dos níveis de grelina (único hormônio orexígeno conhecido, e adipogênico) e uma sinalização precoce do GLP-1 (glucagon - like peptídeo), hormônio capaz de reduzir a velocidade de esvaziamento gástrico, aumentar a secreção de insulina e promover saciedade central, e do PYY (polipeptídio Y), hormônio que diminui a motilidade intestinal e aumenta a saciedade. Tudo isso, pela chegada rápida de alimentos em uma porção mais distal do intestino delgado ${ }^{50}$.

Como efeitos principais, tem-se, portanto, a estimulação precoce do centro da saciedade e a melhora do ciclo da síndrome metabólica. A eficácia da técnica é de $75 \%$ de diminuição do excesso do peso inicial ${ }^{51}$. Suas complicações mais comuns são fístulas, estenoses, úlceras anastomóticas, hérnias internas e embolia pulmonar, que também estão presente nas técnicas predominantemente mal absortivas ${ }^{46,51}$.

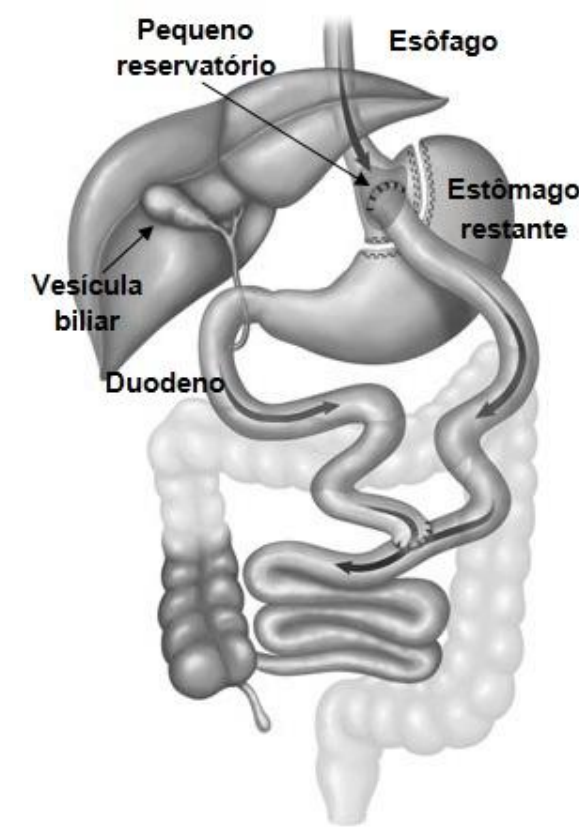

FIGURA 5 - Gastroplastia com derivação gastrojejunal em Y de Roux.

Fonte: (http://www.sbcb.org.br/cbariatrica.asp?menu=1) 
Por fim, o Consenso Bariátrico ${ }^{6}$ entende que os demais procedimentos e técnicas cirúrgicas para o controle da obesidade não relacionados no mesmo, não apresentam indicação atual de utilização ou se encontram em fase de estudos. Tais técnicas carecem de documentações científicas consistentes que permitam sua realização fora de protocolos de pesquisa devidamente regulamentados pelo Conselho Nacional de Ética em Pesquisa (CONEP), em conformidade com a Resolução 196 de 1996 do Conselho Nacional de Saúde ou outra que venha substituí-la ou complementá$\mathrm{la}^{52}$.

Esta revisão apresentou diferentes técnicas cirúrgicas no tratamento da obesidade reconhecidas e recomendadas no mundo inteiro. Pode-se concluir que a escolha da técnica pelo cirurgião irá se basear em informações sobre seu mecanismo de funcionamento, resultados e riscos, disponíveis em relevantes estudos científicos.

\section{REFERÊNCIAS}

1. Coutinho W. Consenso Latino Americano de Obesidade. Arq Bras Endocrinol Metabol. 1999;43:21-67.

2. Mancini MC. Noções Fundamentais - Diagnóstico e Classificação da Obesidade. In: Garrido Júnior AB, Ferraz EM, Barroso FL, et al. Cirurgia da Obesidade. $2^{\underline{a}}$ ed. São Paulo: Atheneu; 2006.

3. Hydock CM. A Brief overview of bariatric surgical procedures currently being used to treat the obese patients. Critical Care Nursing Quarterly. 2005;28(5):217 26.

4. Damaso A, Tock L. Obesidade - perguntas e respostas. Rio de Janeiro: Guanabara Koogan; 2005.

5. Repetto G, Rizzolli J, Bonatto C. Prevalência, riscos e soluções na obesidade e sobrepeso: here, there, and everywhere. Arq Bras Endocrinol Metab. 2003;47(6):6335.

6. Sociedade Brasileira de Cirurgia Bariátrica e Metabólica. Consenso Bariátrico; 2008. [acesso em 2011 Dez 22]. Disponível em: http://www.sbcbm.org.br/ membros_consenso_bariatrico.php

7. Doldi SB, Micheletto G, Di Prisco F et al. Intragastric balloon in obese patients. Obes Surg. 2000;10(6):578-81.

8. Baltasar A, Del Río J, Bengochea M, et al. Cirugía híbrida bariátrica: cruce duodenal en la derivación biliopancreática. Cir Esp. 1996;59:483-6.

9. Scopinaro N, Adami GF, Marinari GM, et al. Biliopancreatic diversion. World J Surg. 1998;22(9):93646.

10. Rabkin RA. Distal gastric bypass and duodenal switch procedure: roux-en-Y gastric bypass and biliopancreatic diversion in a community practice. Obes Surg. 1998;8(1):53-8.

11. Brasil. Resolução CFM oo 1.942 de 5 de fevereiro de 2010. Normas Seguras para Tratamento Cirúrgico da
Obesidade Mórbida. Diário Oficial [da República Federativa do Brasil]. Brasília 12 fev. 2010, Seção I, p. 72.

12. Kuzmak LI. A Review of seven years' experience with silicone gastric banding. Obesity Surg. 1991;1(4):403-8.

13. Kuzmak LI. Stoma adjustable silicone gastric banding. Surgical Rounds. 1991;19-28.

14. Broadbent R, Tracey $M$, Harrington P. Laparoscopic Gastric Banding: a preliminary report. Obes Surg. 1993;3(1):63-7.

15. Belachew $M$, Legrand $M$, Jacquet $N$. Laparoscopic placement of adjustable silicone gastric banding (ASGB) in the treatment of morbid obesity. An animal model experimental study. A video film. A preliminary report [abstract]. Obes Surg. 1993;3:140.

16. Forsell $P$, Hallberg D. Laparoscopic adjustable gastric banding is a safe and effective treatment for morbid obesity. J Soc Laparoendosc Surg. 1998; 2(1):57-61

17. Pitombo C, Jones Jr. KB, Higa KD, et. al. Obesity surgery principles and practice. Nova York: Mc Graw Hill Medical; 2007. p. 101-9.

18. Chapman A, Kiroff G, Game P. Laparascopic adjustable gastric banding in the treatment of obesity: a systematic literature review. Surgery. 2004;135(3):326-51.

19. Zilberstein B, Ferreira JA, Carvalho $M H$, et al. Operação de Scopinaro modificado na falha da banda gástrica. ABCD Arq Bras Cir Dig. 2011;24(2):136-9.

20. Doherty C. Vertical banded gastroplasty. Surg Clin North Am. 2001;81(5):1097-112.

21. DeMaria EJ. Laparoscopic adjustable silicone gastric banding. Surg Clin North Am. 2001;81(5):1129-44,vii.

22. Faintuch J, Matsuda M, Cruz ME, et al. Severe proteincalorie malnutrition after bariatric procedures. Obes Surg. 2004;14(2):175-81.

23. Garrido Júnior $A B$, Ferraz EM, Barroso FL, et al. Cirurgia da Obesidade. $2^{\mathrm{a}}$ ed. São Paulo: Atheneu; 2006. p. 1-7

24. Buchwald H, Avidor $\mathrm{Y}$, Braunwald $\mathrm{E}$, et al. Bariatric surgery: a systematic review and meta-analysis. JAMA. 2004;292(14):1724-37. Erratum in: JAMA. 2005;293(14):1728

25. Marceau P, Biron S, Bourque RA, et al. Biliopancreatic diversion with a new type of gastrectomy. Obes Surg. 1993;3(1):29-35.

26. Milone L, Strong V, Gagner M. Laparoscopic sleeve gastrectomy is superior to endoscopic intragastric balloon as a first stage procedure for super-obese patients (BMI $\geq 50$ ). Obes Surg. 2005;15(5):612-7.

27. Regan JP, Inabnet WB, Gagner M. Early experience with two-stage laparoscopic Roux-en- $Y$ gastric bypass as an alternative in the super-super obese patient. Obes Surg. 2003;13(6):861-4.

28. Almogy G, Crookes PF, Anthone G. Longitudinal gastrectomy as a treatment for the high-risk superobese patient. Obes Surg. 2004;14(4):492-7.

29. Langer FB, Reza Hoda MA, Bohdjalian A, et al. Sleeve gastrectomy and gastric banding: effects on plasma ghrelin levels. Obes. Surg. 2005;15(7):1024-29.

30. Hamoui N, Anthone GJ, Kaufman HS, et al. Sleeve gastrectomy in the high-risk patient. Obes Surg. 2006;16(11):1445-49.

31. Lalor PF, Tucker ON, Szomstein S, et al. Complications after laparoscopic sleeve gastrectomy. Surg Obes Relat Dis. 2008;4(1):33-8.

32. Brethauer SA, Hammel JP, Schauer PR. Systematic review of sleeve gastrectomy as staging and primary bariatric procedure. Surg. Obes Relat Dis. 2009;5(4):46975 . 
33. Lee CM, Cirangle PT, Jossart GH. Vertical gastrectomy for morbid obesity in 216 patients: report of two-year results. Surg Endosc. 2007;21(10):1810-6.

34. O'Brien PE. Bariatric surgery: mechanisms, indications and outcomes. J Gastroenterol Hepatol. 2010;25(8):135865.

35. Almeida N, Gomes D, Gonçalves C, et al. O balão intragástrico nas formas graves de obesidade. GE - J Port Gastrenterol. 2006;13:220-5.

36. Galloro G, De Palma GD, Catanzano C, et al. Preliminary endoscopic technical report of a new silicone intragastric balloon in the treatment of morbid obesity. Obes Surg. 1999;9(1):68-71.

37. Genco A, Bruni T, Doldi SB, et al. Bioenterics intragastric balloon: the Italian experience with 2515 patients. Obes Surg. 2005;15(8):1161-4.

38. De Waele B, Reynaert H, Urbain D, et al. Intragastric balloons for preoperative weight reduction. Obes Surg. 2000;10(1):58-60.

39. Scopinaro N, Adami GF, Marinari GM, et al. Biliopancreatic diversion: two decades of experience. In: Deitel M, Cowan SM, et al. Update: surgery for the morbidly obese patient. Toronto, Canada: FDCommunications Inc; 2000:227-58.

40. Fruhbeck G, Diez-Caballero A, Gil MJ, et al. The decrease in plasma ghrelin concentrations following bariatric surgery depends on the functional integrity of the fundus. Obes Surg. 2004:14(5):606-2.

41. Conselho Regional de Medicina de Pernambuco. Redução de estômago não será só para obesos. [acesso em 2011 dez. 22]. Disponível em: http://www.cremepe.org.br/ leitorClipping.php?cd_clipping $=6314$

42. Hess DS, Hess DW. Biliopancreatic diversion with a duodenal switch. Obes Surg. 1998;8(3):267-2.

43. Hess DS, Hess DW, Oakley RS. The biliopancreatic diversion with the duodenal switch: results beyond 10 years. Obes Surg. 2005;15(3):408-16.

44. Gedea ME, Arribas del Amo D, Solanas JA, et al. Results of biliopancreatic diversion after five years. Obes Surg. 2004;14(6):766-81.

45. Rabkin RA, Rabkin JM, Metcalf B, et al. Laparoscopic technique for performing duodenal switch with gastric reduction. Obes Surg. 2003;13(2):263-8.

46. Maggard MA, Shugarman LR, Suttorp M, et al. Metaanalysis: surgical treatment of obesity. Ann Intern Med. 2005;142(7):547-9.

47. Garrido Júnior AB. Cirurgia em obesos mórbidos: experiência pessoal. Arq Bras Endocrinol Metabol. 2000;44:106-13.

48. Westling A, Gustavsson S. Laparoscopic vs open Rouxen-Y gastric bypass: a prospective, randomized trial. Obes Surg. 2001;11(3):284-92.

49. Balsiger BM, Kennedy FP, Abu-Lebdeh $\mathrm{HS}$, et al. Prospective evaluation of Roux-en- $Y$ gastric bypass as primary operation for medically complicated obesity. Mayo Clin Proc. 2000;75(7):673-80.

50. Korner J., et al. Effects of Roux-en-Y gastric bypass surgery on fasting and postprandial concentrations of plasma ghrelin, peptide $Y Y$, and insulin. J. Clin. Endocrinol Metab. 2005;90(1):359-65.

51. Santry HP, Gillen DL, Lauderdale DS. Trends in bariatric surgical procedures. JAMA. 2005;294(15):1909-17.

52. Ministério da Saúde. Resolução CNS no 196 de 10 de outubro de 1996. Diretrizes e normas regulamentadoras de pesquisas envolvendo seres humanos. [resolução na internet]. [acesso em 2011 set. 12]. Disponível em: http://conselho.saude.gov.br/web_comissoes/conep/aquiv os/resolucoes/resolucoes.htm

\section{Endereço para correspondência:}

Poliana Oliveira Novais

Quadra 203 Norte Al.2 Qi.B Lt.6

Palmas/TO - CEP 77006-880

Telefone: +556381314177

E-mail:plinovais@hotmail.com 